\title{
Self-Induced Trauma from Compulsive Parafunction
}

\author{
Lagcher $0^{\prime}$ Brien $\mathrm{SM}^{1}$ and Hasan $\mathrm{I}^{2 *}$ \\ ${ }^{1} D 3$ Student, ECU School of Dental Medicine, USA \\ ${ }^{2}$ Assistant Professor, Oral Medicine, Dept of Surgical Sciences, ECU School of Dental Medicine, USA
}

*Corresponding author: Hasan I, Assistant Professor, Oral Medicine, Dept of Surgical Sciences, ECU School of Dental Medicine, USA
Received Date: June 11, 2020

Published Date: June 29, 2020

\section{Summary}

A 32-year-old female presented with a chronic, localized pain from a described burning sensation in the mouth. Primary burning mouth syndrome (BMS) was initially diagnosed and the patient was treated with pharmacological therapies. The neuropathic oral pain was later found to be actualizing from a compulsive parafunctional habit. This case-report aims to emphasize proper diagnosis through additional means of evaluation and utilizing readily available diagnostic tools prior to initiating treatments.

\section{Background}

Neuropathic pain is a complex condition initiated by perturbation of the nervous system from lesion, injury or disease [1-3]. It may be related to either or both peripheral and central nervous system disorders [1-4]. Current estimates for neuropathic pain prevalence in the general population range from $6.9-10 \%$, with $7-8 \%$ most frequently cited in literature $[1,2,5]$. Prevalence estimates are expected to increase due to ageing populations, obesity rates, anxiety-related disorders, incidence of diabetes mellitus, and improved survival outcomes of cancer patients post chemotherapy [1,5]. Neuropathic pain may be distinguished as spontaneous or evoked [1,2]. It can be accompanied by paresthesia, dysesthesia, anesthesia or sensations of weakness in associated areas [2,3].

Causes of neuropathic pain can be difficult to identify and manage. Etiology has been linked to trauma, chemotherapy, amputation, nerve compression, post-herpetic neuralgia, stroke, inflammatory disorders, autoimmune disorders and immunocompromising conditions [1,3,4]. Neuropathic orofacial pain can manifest as atypical odontalgia post extraction, trigeminal neuralgia or BMS $[3,6,7]$. Due to numerous etiologies, multiple attempts to ascertain underlying causes may need to be pursued. It is additionally imperative to assess the severity of the pain while considering individual comorbidities and the overall impact on a person's work, social and personal life [1].

Keywords: Neuropathic pain; Idiopathic pain; Burning mouth syndrome; Parafunctional habit; Self-induced trauma; Compulsive behavior

\section{Case Presentation}

A 32-year-old Caucasian female presented to clinic with complaint of a chronic burning sensation localized to the anterior tongue tip. Symptom was determined to exceed six months. Patient medical history disclosed hyperthyroidism, type 2 diabetes mellitus and mild obsessive-compulsive disorder (OCD). Medications were limited to methimazole and metformin at the time of initial consultation. No significant family or social history was identified.
Neurology had previously diagnosed primary BMS based on the exclusion of other conditions and determining the pain as idiopathic. The patient was symptomatically treated with pharmacological therapies. She was initially prescribed gabapentin and reported a side-effect of excessive dizziness with consumption. Gabapentin was alternatively replaced with amitriptyline which caused her extended drowsiness. Although the patient reported treatments being effective in symptom reduction, she chose to discontinue 
pharmacological therapy due to side-effects and seek care with oral medicine.

Significant clinical findings or abnormalities were not detected during initial examination with oral medicine. Intraoral photographs were obtained for documentation and further review of the patient's oral condition. The high magnification and resolution photographs revealed patched papilla balding and non-obvious tooth indentations outlined on the right anterior tongue tip. The patient had initially denied oral habits that could be linked as a source of pain. After further questioning and reviewing photographic findings, she acknowledged her husband had repeatedly noticed her biting the tip of her tongue. The patient's husband accompanied her to scheduled appointments and confirmed the habit while describing it as a subconscious compulsion likely related to her history of OCD. A working diagnosis was made to include self-inflicted trauma from a compulsive oral parafunctional habit (Figure 1).

Figure 1

\section{Treatment}

Current literature supports first-line pharmacological management of neuropathic pain with anti-convulsant medications and tri-cyclic antidepressants $[1,2,5,8]$. Topical medications such as lidocaine or capsaicin in patch form have also been used in some instances $[1,2,5,8]$. One study cited use of a topical lubricant containing glycerin in carboxymethylcellulose (CMC) solution as a treatment strategy for pain related symptoms. 9 Little success has been documented in using non-steroidal anti-inflammatory drugs (NSAIDs) to manage neuropathic pains, and opioids have weak recommendations for use due to long-term side-effects and potential addiction risk $[1,2,8]$. Other research includes nonpharmacological management with occlusal splint therapy, and behavior modification through self-adjustment or counseling [9-11]. There is a concurrence throughout available literature on the need to also consider and address psychogenic factors when treating neuropathic pain. Stress and personality may participate in individual pain presentations and reported symptom severity.

Nonpharmacological behavior modification was the recommended treatment method in this case. It included a mobile device cessation alarm repeated every thirty minutes of waking hours, and a mandibular occlusal splint. The patient reported a 40-50\% improvement in symptoms at 6-week follow-up. Pain was described as absent at 18-week follow-up. Subsequent follow-ups were recommended as needed and the patient was discharged from clinic.

\section{Conclusion}

Self-induced trauma from parafunctional habit should be included as a differential diagnosis of neuropathic oral pain in the absence of pathology. Comprehensive interviews, clinical examinations, laboratory investigation, high-definition photographs and imaging modalities are prudent prior to definitive or irreversible treatments. This case indicates trauma signs may be so discreet that they are not clinically noticeable and are easily missed. Dentists should be competent in the identification, diagnosis and management of these habits, and their relation to some neuropathic oral pains.

\section{Acknowledgement}

None.

\section{Conflict of Interest}

No conflict of interest.

\section{References}

1. Colloca L, Ludman T, Bouhassira D, Ralf Baron, Anthony H Dickenson, et al. (2017) Neuropathic pain. Nat Rev Dis Primers 3:17002.

2. Gilron I, Baron R, Jensen T (2015) Neuropathic pain: Principles of Diagnosis and Treatment. Mayo Clinic Pro 90(4): 532-545. 
3. Tinastepe N, Oral K (2013) Neuropathic pain after dental treatment. Agri 25(1): 1-6.

4. Costigan M, Scholz J, Woolf CJ (2009) Neuropathic Pain: A Maladaptive Response of the Nervous System to Damage. Annu Rev Neurosci. 32: $1-32$.

5. Cruccu G, Truini A (2017) A review of Neuropathic Pain: From Guidelines to Clinical Practice. Pain and Ther 6(Suppl 1): 35-42.

6. Romero-Reyes M, Uyanik JM (2014) Orofacial pain management: current perspectives. J Pain Res 7: 99-115.

7. Jääskeläinen SK (2018) Is burning mouth syndrome a neuropathic pain condition? Pain 159(3): 610-613.

8. Dworkin RH, O'Connor AB, Audette J, Ralf Baron, Geoffrey K Gourlay, et al. (2010) Recommendations for the Pharmacological Management of
Neuropathic Pain: An Overview and Literature Update. Mayo Clin Pro 85(3 Suppl): S3-S14.

9. Kho HS, Lee JS, Lee EJ, Lee JY (2010) The effects of parafunctional habit control and topical lubricant on discomforts associated with burning mouth syndrome (BMS). Arch Gerontol Geriatr 51(1): 95-99.

10. Cannavale R, Itro A, Campisi G, Compilato D, Colella G (2015) Oral self-injuries: Clinical findings in a series of 19 patients. Med Oral Patol Oral Cir Bucal 20(2): e123-e129.

11. Glaros AG, Owais Z, Lausten L (2006) Reduction in parafunctional activity: a potential mechanism for the effectiveness of splint therapy. J Oral Rehabil 34: 97-104. 